\title{
Gordailu, Centro de Patrimonio Cultural Mueble de Gipuzkoa. Estudios previos
}

\author{
Maite Barrio Olano, Ion Berasain Salvarredi
}

Resumen: La Diputación Foral de Gipuzkoa ha impulsado la creación de una nueva infraestructura cultural: el Centro de Patrimonio Cultural Mueble de Gipuzkoa, denominado Gordailu (palabra en euskera cuyo significado es “depósito”). Este Centro tendrá como misión la gestión integral del patrimonio cultural mueble del territorio, partiendo de un núcleo central, el depósito in situ de colecciones de distinta titularidad. Dentro de la variedad de temas de estudio y análisis que implica un proyecto de esta envergadura, en el presente artículo se relatan los relativos al plan de necesidades, evaluación de colecciones y definición de condiciones medioambientales.

Palabras clave: Depósitos, gestión patrimonio, conservación preventiva, evaluación colecciones.

Abstract: The Provincial Government of Gipuzkoa (Diputación Foral de Gipuzkoa) has given stimulus to the creation of a new cultural facility: the Centre for the Cultural Heritage of Gipuzkoa which will be called Gordailu (Wharehouse in basque language). This Centre will have as its goal the comprehensive management of the Cultural Heritage of the Province, operating from a coordinating centre, with on-site storage for the various and distinct collections. This present article relates to the overall strategy, the evaluation of collections and the necessary micro-climatic specificattions for storage required within the wide range of themes and analysis which the scope of such an ambitious project implies.

Keywords: Storage, management Cultural Heritage, Preventive Conservation, evaluation of collections

Resumo: A Diputación Foral de Gipuzkooa tem impulsado a criação de uma nova infra-estructura cultural: o Centro de Patrimonio Cultural Mueble de Gipuそkoa, denominado Gordailu (palavra em euskera cujo significado é “depósito"). Este centro terá como missão a gestão integral do património cultural móvel do território, partindo de um núcleo central: o depósito in situ de colecções de distinta titularidade. Dentro da variedade de temas de estudo e análise que implica um projecto desta envergadura, no presente artigo relatam-se os relativos ao plano de necessidades, avaliação de colecções e definição de condições medio-ambientais.

Palavras-chave: Depósitos, gestão património, conservação preventiva, avaliação, colecções.

\section{Gordailu, Centro de Patrimonio Cultural Mueble}

El Departamento de Cultura y Euskera de la Diputación Foral de Gipuzkoa ha impulsado la creación de una nueva infraestructura cultural: el Centro de Patrimonio Cultural Mueble de Gipuzkoa, denominado Gordailu. Este Centro tendrá como misión la gestión integral del patrimonio cultural mueble del territorio, partiendo de un núcleo central, el depósito in situ de colecciones de distinta titularidad. Estas serán:

- Colección de Diputación Foral de Gipuzkoa, hoy en día dispersa en varios almacenes, con un número aproximado de 24.500 piezas de etnografía y bellas artes principalmente.

- Colección del Museo San Telmo, perteneciente al Ayuntamiento de Donostia-San Sebastián. Comprende bienes de arqueología, bellas artes, etnografía e historia (12.840 piezas aprox.). Actualmente el museo se encuentra en un periodo de renovación y 
ampliación, y Gordailu permitirá la liberación de espacios anteriormente dedicados a almacenaje para nuevos usos de exposición u otras disposiciones museológicas.

- Posibilidad de incorporación del Depósito Arqueológico de Gipuzkoa, con material principalmente cerámico, lítico y orgánico, gestionado por el Gobierno Vasco.

- Los depósitos estarán abiertos al almacenaje de otros bienes culturales muebles, pertenecientes a colecciones públicas o privadas de interés cultural del entorno. Este es el caso la Caja de Ahorros y Monte de Piedad de Guipúzcoa y San Sebastián "KUTXA" que utilizará esta infraestructura como depósito de sus colecciones.

La vocación de Gordailu es la de ofrecer los servicios oportunos al conjunto de las instituciones públicas y privadas así como a particulares del Territorio Histórico de Gipuzkoa, en temas de patrimonio cultural mueble, garantizando y promoviendo el conocimiento, la conservación y la difusión del mismo. Será, además, el corazón y el motor del renovado sistema de museos del territorio, una de las principales apuestas estratégicas del departamento en materia cultural, mediante la creación de colecciones itinerantes, servicio de depósito, préstamo y asesoramiento técnico.

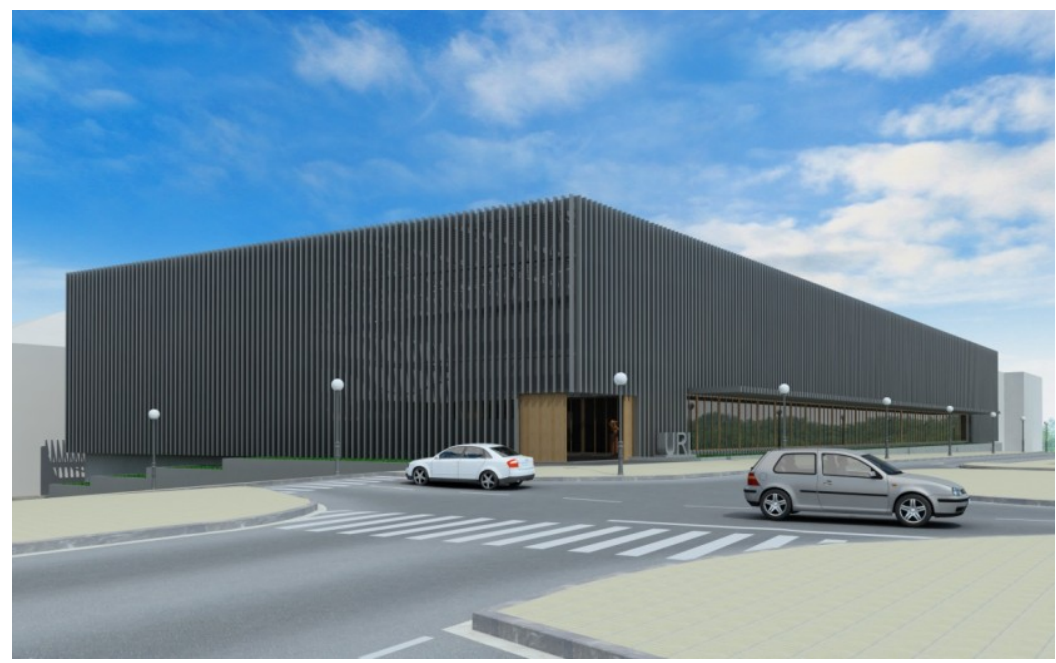

Figura 1: Vista exterior del Centro. Infografía. Astigarraga y Lasarte arquitectos.

Actualmente, el Centro de Patrimonio se encuentra en fase de construcción en el solar que ocupaba el antiguo pabellón industrial URI, de Irún. El edificio, de tres plantas y $9.116 \mathrm{~m}^{2}$, obra del estudio de arquitectura Astigarraga y Lasarte de San Sebastián, será un bloque básicamente compacto con una envolvente exterior de lamas, específicamente diseñado en función de las necesidades de conservación de los materiales. Para garantizar su cometido, los espacios han sido diseñados de manera que faciliten el conjunto de operaciones necesarias para la conservación, entendiendo esta en su sentido más amplio. Además, la trazabilidad de flujos y circulaciones posibilitará el acceso público, favoreciendo una política de difusión y conocimiento del patrimonio

En el presente artículo pretendemos dar a conocer una parte de los estudios previos realizados a fin de poder materializar en un proyecto arquitectónico las necesidades de un centro de las características de Gordailu, que cubre el ciclo completo de gestión de un bien cultural mueble: desde su depósito, registro, conservación preventiva, restauración, control y análisis de riesgos, hasta su investigación y difusión, bien sea in situ o derivada hacia otras infraestructuras de exposición. 
El complejo trabajo de diseño, definición y puesta a punto de este proyecto ha sido realizado por técnicos del Servicio de Patrimonio Histórico-Artístico, Archivos y Museos de la Dirección General de Patrimonio Cultural gipuzcoano. También ha sido precisa la experta colaboración de los departamentos de Movilidad y Ordenación del Territorio, así como del de Presidencia y Administración Foral. No podemos olvidar tampoco la valiosa colaboración de los técnicos del donostiarra Museo de San Telmo, así como del Centro de Patrimonio Cultural del Gobierno Vasco.

El concepto de este depósito difiere profundamente de la idea de almacén tradicional, en el que se guardaban los objetos que no podían ser expuestos. Por el contrario, aborda una concepción dinámica de la custodia y gestión de colecciones, en estrecha relación con los nuevos intereses en conservación preventiva de los bienes culturales.

\section{Planificación del nuevo Centro}

La complejidad de un proyecto de esta naturaleza obliga al análisis previo de numerosas cuestiones, muchas de ellas forzosamente relacionadas entre sí. Por ello, desde 2005 se han llevado a cabo diversos estudios, desde el propio concepto de Centro de Patrimonio - con especificación y descripción de las actividades a desarrollar y espacios y equipamientos necesarios para ello-, hasta el análisis de colecciones (características, estado de conservación, inventarios, bases de datos, etc.), o parámetros de conservación, climatología, seguridad, evacuación de residuos, etc.

En el presente artículo nos centramos concretamente en algunos de los aspectos estudiados:

1. Plan de necesidades.

2. Evaluación de colecciones.

3. Definición de condiciones medioambientales.

1. Plan de necesidades ${ }^{1}$

Tal y como hemos enunciado, Gordailu se presenta como un Centro de carácter plurifuncional, cuyos espacios deberán albergar y facilitar la consecución de diversas prácticas y usos. Por ello, en un primer momento, se planteó como imprescindible la detección y definición de estas actividades, y el diseño de su funcionamiento, con la secuencia lógica de flujos y circulaciones que implicaban, así como el requerimiento espacial de equipamientos e instalaciones.

\section{Espacios}

Los espacios determinados fueron los siguientes²:

- Áreas de acceso restringido:

\section{A/ Servicios técnicos}

- Muelle de carga y descarga. Prepara la recepción del medio de transporte por carretera, proporcionando un espacio a cubierto para la carga y descarga.

- Espacio de embalaje y desembalaje. Disponible para retirar o colocar elementos de protección para el transporte y facilitar una primera inspección visual del objeto.

- Registro. Además del registro y control de las piezas, podrán realizarse trabajos de acondicionamiento de los objetos para su correcto almacenaje. 
- Estudio de fotografía. Destinado a la documentación fotográfica, reflectográfica y radiográfica de los bienes custodiados, para inventarios, catálogos, publicaciones, peticiones y procesos de intervención.

- Almacén de tránsito. Compartimentado, próximo a la zona de carga y descarga, cumple la función de distribución de recepciones y/o envíos.

-5. USOS DE ESPACIOS Y PREVISIÓN DE SUPERFICIES'.
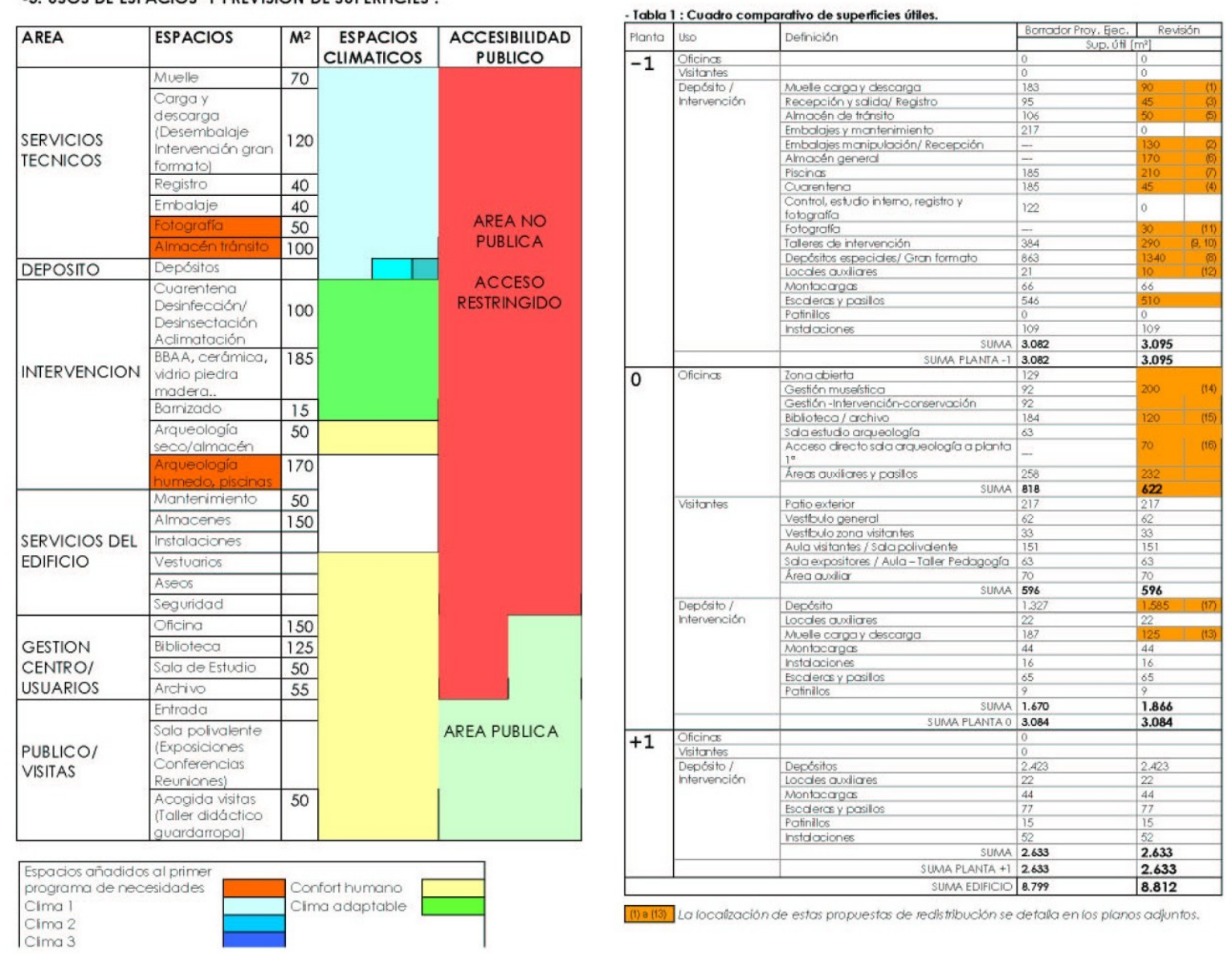

Figura 2: Uso de espacios y previsión de superficies. Esquema general y desarrollo por plantas.

\section{B/ Área de Intervención}

Cuenta con cuatro espacios diferenciados en función de la intervención a efectuar. Está en relación directa con el área de servicios técnicos y es el paso preliminar al almacenaje de aquellos objetos que requieren algún tratamiento. Igualmente, será el lugar de conservación y/o restauración de aquellos objetos almacenados que precisen algún tipo de mantenimiento, prestando el mismo servicio para otros elementos provenientes del Territorio que requieran esta instalación especializada.

- Espacio de cuarentena /desinsectación / aclimatación: Se trata de un espacio plurifuncional, donde las acciones se programarán para evitar interferencias entre aquellas que no sean compatibles. La aclimatación, en algunas ocasiones, podrá también llevarse a cabo en el almacén de tránsito o en la zona de embalaje.

- Taller de conservación y restauración: Con excepción de materiales arqueológicos. El espacio puede ser subdividido de forma sencilla en el caso de que se realicen simultáneamente actividades incompatibles. 
- Talleres de restauración y conservación de material arqueológico: Diferenciándose los espacios según sean tratamientos acuosos o en seco. En el primer caso, se cuenta con una gran piscina para desalinización de objetos de gran volumen, directamente conectada con el muelle de carga mediante una grúa puente. Así mismo se cuenta con zonas específicas para limpieza de material y depósitos de diferentes tamaños para desalinización de objetos.

- Espacio de liofilización y cámaras frigoríficas.

\section{C/ Depósitos}

Los depósitos quedan diferenciados en dos categorías en función del volumen y masa de los objetos que van a albergar:

- Objetos de gran formato: aquellos que cumplen cualquiera de estas dos condiciones: una de sus tres dimensiones supera los 2 metros o su peso excede de los $100 \mathrm{~kg}$.

- Objetos de pequeño y mediano formato: el resto ${ }^{3}$.

Dentro de esta área se delimita una zona específica destinada a material arqueológico, caracterizado por su crecimiento constante, su almacenamiento en contenedores estandarizados y por la frecuencia de su consulta por parte de investigadores.

Así mismo se prevé un almacén de alta seguridad para aquellos bienes que por su especial valor o importancia requieran un control específico.

D/ Servicios del edificio

Zonas destinadas a instalaciones, mantenimiento, vestuarios, aseos, seguridad, almacenes de material, recogida de residuos, etc.

- Áreas públicas:

A/ Espacios destinados a gestión del propio Centro y consulta e investigación

Oficinas para las diferentes actividades que se desarrollan en el centro, Centro de documentación sobre temas relativos al patrimonio cultural mueble y Sala de Investigadores, destinada a los usuarios que requieran el estudio de piezas depositadas.

B/ Salas de difusión

La infraestructura se completa con una sala polivalente y un aula didáctica, donde llevar a cabo conferencias, proyecciones, seminarios, cursos, reuniones, exposiciones, talleres, acogida de visitantes, etc.

Una vez determinados las actividades a realizar y sus espacios, se ha profundizado en la definición del carácter específico de cada uno de ellos y en el diseño de funcionamiento. Así, y a modo de ejemplo, en el caso del centro de documentación se han estudiado los diferentes recursos existentes en Gipuzkoa en relación al patrimonio histórico y el tipo de usuario al que va dirigido, con la finalidad de evitar duplicidades y centrarse en aquellos aspectos en los que se observan carencias.

Tras analizar estos datos en relación a los principios básicos que regirán el trabajo del centro y en coordinación con los técnicos de la administración, se han revisado las necesidades y requisitos necesarios de cada uno de los espacios estudiados, y todas estas especificaciones han sido detalladas en fichas por sectores. 

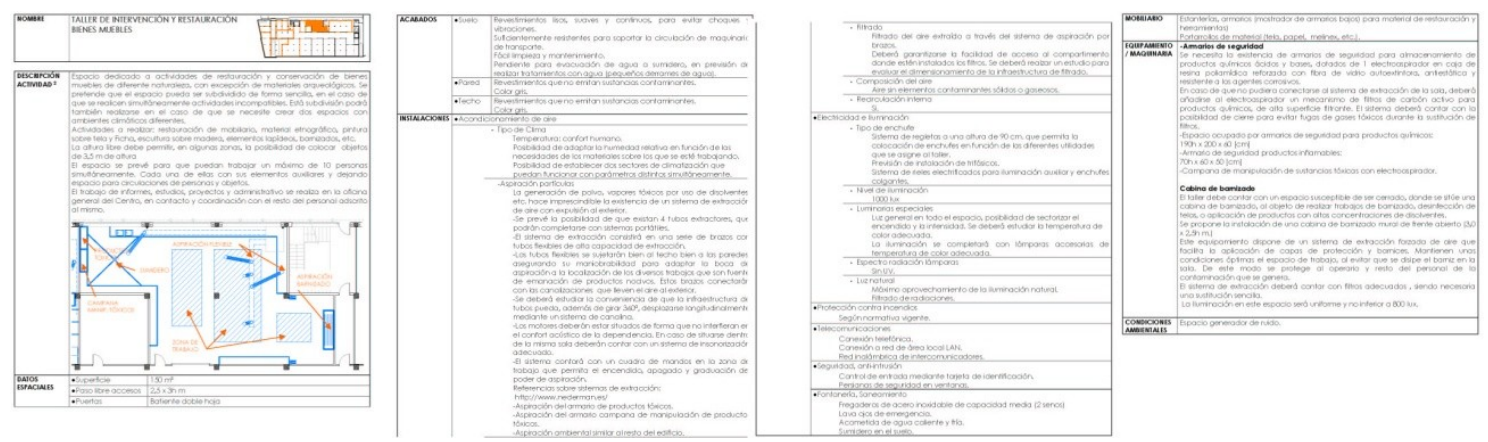

Figura 3: Ficha técnica del taller de conservación y restauración.

Los datos reflejados en el documento hacen referencia a:

- Descripción de la actividad que se va a realizar en dicho espacio.

- Datos espaciales: superficie requerida, paso libre de accesos, tipo de puertas.

- Acabados de suelo, pared y techo.

- Instalaciones:

- Acondicionamiento de aire (consignas climáticas requeridas, sectorizaciones, aspiración de partículas y vapores tanto a nivel general como de equipamientos específicos, filtrado, recirculaciones, etc...)

- Electricidad e iluminación (tipo de conexiones, potencias, niveles de iluminación, características de la misma).

- Protección contra incendios

- Voz y datos.

- Seguridad.

- Fontanería, saneamiento.

- Mobiliario.

- Maquinara.

\section{Circulaciones}

Resulta evidente que para un desarrollo eficaz de la actividad del Centro, sea necesario agrupar los espacios y diseñar las circulaciones con el objetivo de crear las conexiones funcionales oportunas. No debemos olvidar un factor de complejidad específico, que viene dado por la coexistencia en el mismo edificio de espacios dedicados al público y otros de acceso restringido, restricción determinada bien sea por la seguridad de los propios bienes culturales custodiados o por la ejecución de trabajos por parte del personal del Centro.

Estas conexiones posibilitan en ocasiones la existencia de espacios lo suficientemente flexibles como para que se puedan desarrollar actividades en coordinación con espacios adyacentes. Es el caso del espacio de embalaje y desembalaje, núcleo alrededor del cual se distribuyen los servicios técnicos que acompañan el ingreso y salida de los bienes guardados en el depósito. A pesar de que su cometido principal consiste en la preparación para el transporte y el acondicionamiento para el almacenaje de las piezas, este espacio puede utilizarse también, en caso necesario, como zona de registro -si el espacio previsto para el mismo resulta insuficiente-, o para intervenciones de conservación y restauración puntuales de objetos de gran volumen e incluso como plató fotográfico para los mismos. De esta misma forma, el muelle puede utilizarse para la limpieza con sistemas acuosos de objetos de gran tamaño que así lo requieran. 
Se han definido dos tipos principales de circulación:

$1^{\circ}$ Circulación de objetos:

- siguiendo una secuencia lógica de entrada o salida del Centro, incluyendo tanto las piezas destinadas a su almacenamiento, como aquellas asignadas únicamente a un tratamiento de conservación-restauración.

- en el interior del edificio, de cara a intervenciones, adecuación de sistemas de almacenamiento, exposiciones o estudio en la sala de investigación.

Para evaluar las posibilidades de movimiento de las piezas, ha sido necesario considerar tanto el volumen como la masa de las mismas, realizando simulaciones de manipulación de los objetos más desfavorables sobre el plano.

$2^{\circ}$ Circulación de personas:

- Aquellas que desarrollen su trabajo en el Centro, sea personal adscrito al mismo, contratas de servicios puntuales, contratas de mantenimiento, suministros, etc.

- Público visitante, sea por visitas a la propia infraestructura, sea por actividades programadas -talleres, conferencias exposiciones, etc.-.

- Investigadores y estudiosos, así como usuarios de servicios tales como el Centro de documentación, sala de investigación, etc.

Se han estipulado áreas que puedan ser independientes en función de la actividad y horario que van a desarrollar4.

Las circulaciones verticales en el edificio se realizan a través de un núcleo de escaleras principal (que puede complementarse por motivos de servicio con otros dos núcleos de escaleras, necesarios en cualquier caso por motivos de evacuación en caso de emergencia), dos montacargas y un ascensor.

$2^{\circ}$ Evaluación espacial de las colecciones.

El diseño de los depósitos, tanto a nivel de volumen requerido como de condiciones y mobiliario de almacenamiento, exige un análisis de las colecciones desde diversos parámetros.

La naturaleza heterogénea de los objetos a albergar en Gordailu hizo necesario un ejercicio de síntesis, para hacer confluir la variedad de dimensiones (unida a la casuística de materiales y condiciones de conservación), con una necesaria estandarización del soporte de almacenaje.

El punto de partida para realizar dicho estudio fueron los diferentes inventarios y bases de datos existentes de las colecciones, que recogían información de más de 35.000 objetos. Sus características y alcance variaban en función de la institución responsable de su custodia.

Mediante una migración de los datos disponibles a una base única y posteriormente a una hoja de cálculo se llevó a cabo una agrupación por:

-Materiales: cada uno de los materiales demanda un tipo de condición climática determinada.

-Tipo de objeto: cada familia de objetos requiere un mobiliario de almacenamiento específico. 
-Dimensiones reales del objeto: se adoptan unos márgenes de manipulación, transformando el volumen real del objeto, sea cual sea su forma, en formas cúbicas envolventes, pues los objetos, en función de sus medidas de volumen y masa, precisan un tipo de almacenamiento determinado.

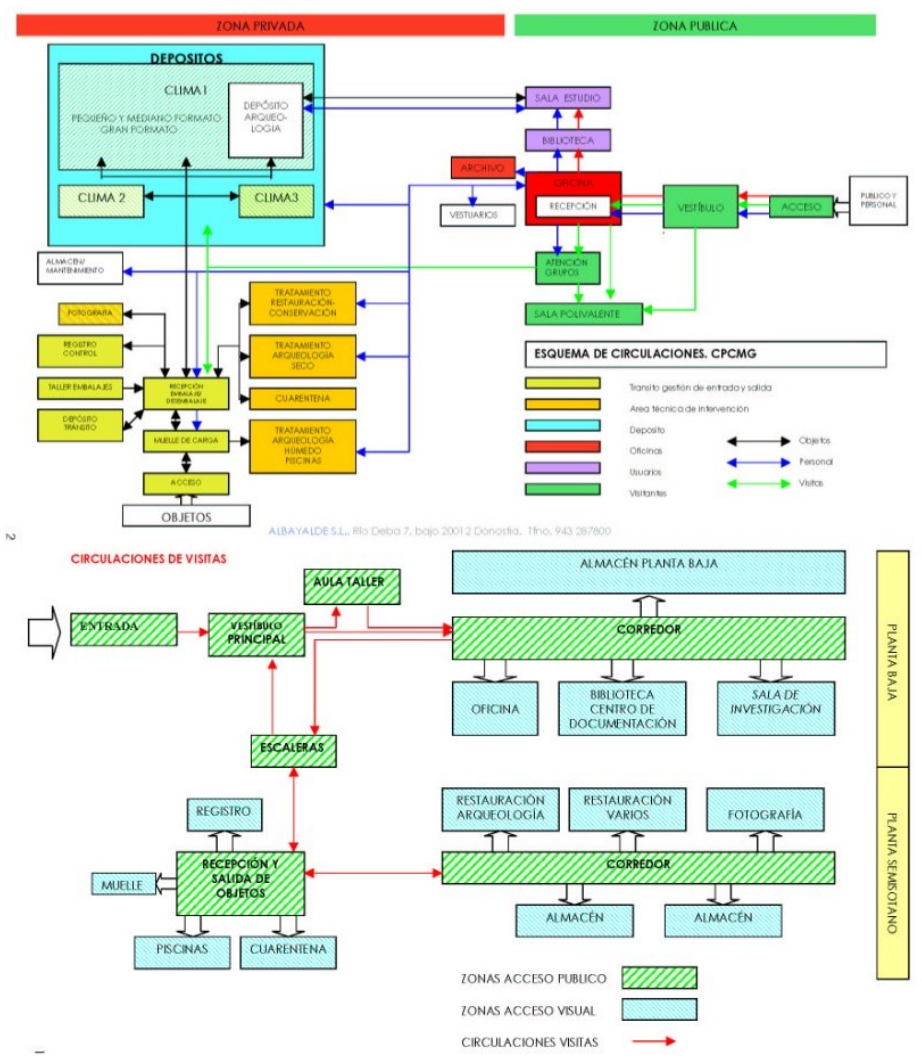

Figura 4: Esquemas de circulaciones.

Estos parámetros propios del objeto (material, tipo y dimensión) condicionan su ubicación dentro del depósito. El material está relacionado con la categoría climática; el tipo de objeto con la agrupación por denominación; la dimensión del objeto condiciona sus condiciones de manipulación ya sea por medios humanos o mecánicos. La combinación de todos ellos, da lugar a una compleja distribución dentro del sistema de almacenaje.

De esta manera se perseguía la obtención de los siguientes datos:

- Volúmenes de objetos y su ocupación en los diferentes sistemas de almacenamiento.

- Selección y cuantificación del mobiliario necesario para albergar los objetos.

- Distribución de volúmenes y superficies en función de las necesidades climáticas de los materiales.

- Distribución del mobiliario en los depósitos, evitando posibles interferencias con las instalaciones del edificio (conducciones de climatización, iluminación, accesos, etc.), optimizando circulaciones y ocupación.

Sin embargo, la migración reveló la existencia de ciertas carencias, de mayor o menor importancia, en cuanto a contenidos relativos a materiales, estado de conservación, tipo de objeto etc., así como cierta confusión en la recogida de información (medidas en diferentes unidades, diversa nomenclatura para el mismo tipo de objeto, etc.). 
Ante las desviaciones de información causadas por estas lagunas e insuficiencias en los datos previos, se juzgó preferible un cambio de metodología, en aras a obtener una información más real sobre la naturaleza y la previsible ocupación de las colecciones. De esta manera se procedió a la visita de los almacenes de las tres instituciones, analizando mediante trabajo de campo las 7 localizaciones de almacenaje (superficie conjunta aprox. $3.644 \mathrm{~m}^{2}$ ), en planta y las obras expuestas en las diferentes salas del Museo de San Telmo.

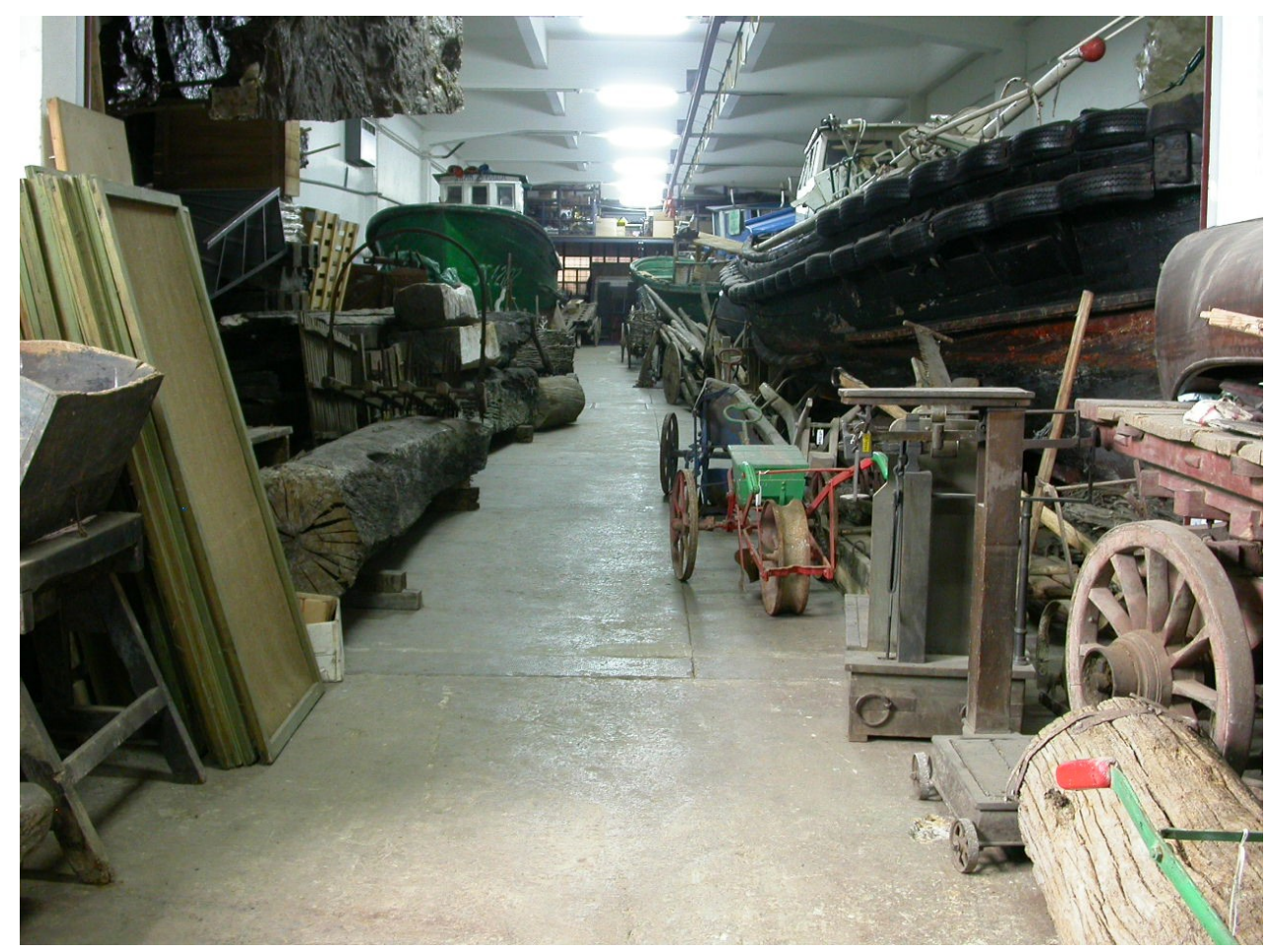

Figura 5: Imagen de los actuales depósitos de DFG.

Durante este sondeo se llevaron a cabo los siguientes estudios:

-Mobiliario de almacenamiento

-Medición de módulos y cuantificación de los mismos.

-Cuantificación del nivel de ocupación de los estantes.

-Extrapolación de la ocupación de estantes a un nivel de ocupación adecuado.

-Estimación espacial de los objetos existentes depositados fuera de muebles de almacenamiento ${ }^{5}$ (un total de 5.272 piezas).

La información obtenida fue ordenada adecuadamente mediante formularios, posteriormente volcados a hojas de cálculo, en las que se incluye:

- Medidas de los objetos (alto, ancho, fondo).

- Tipo de objeto.

- Número de objetos similares.

- Grupo climático adscrito.

- Lugar de almacenamiento.

- Referencias fotográficas. 


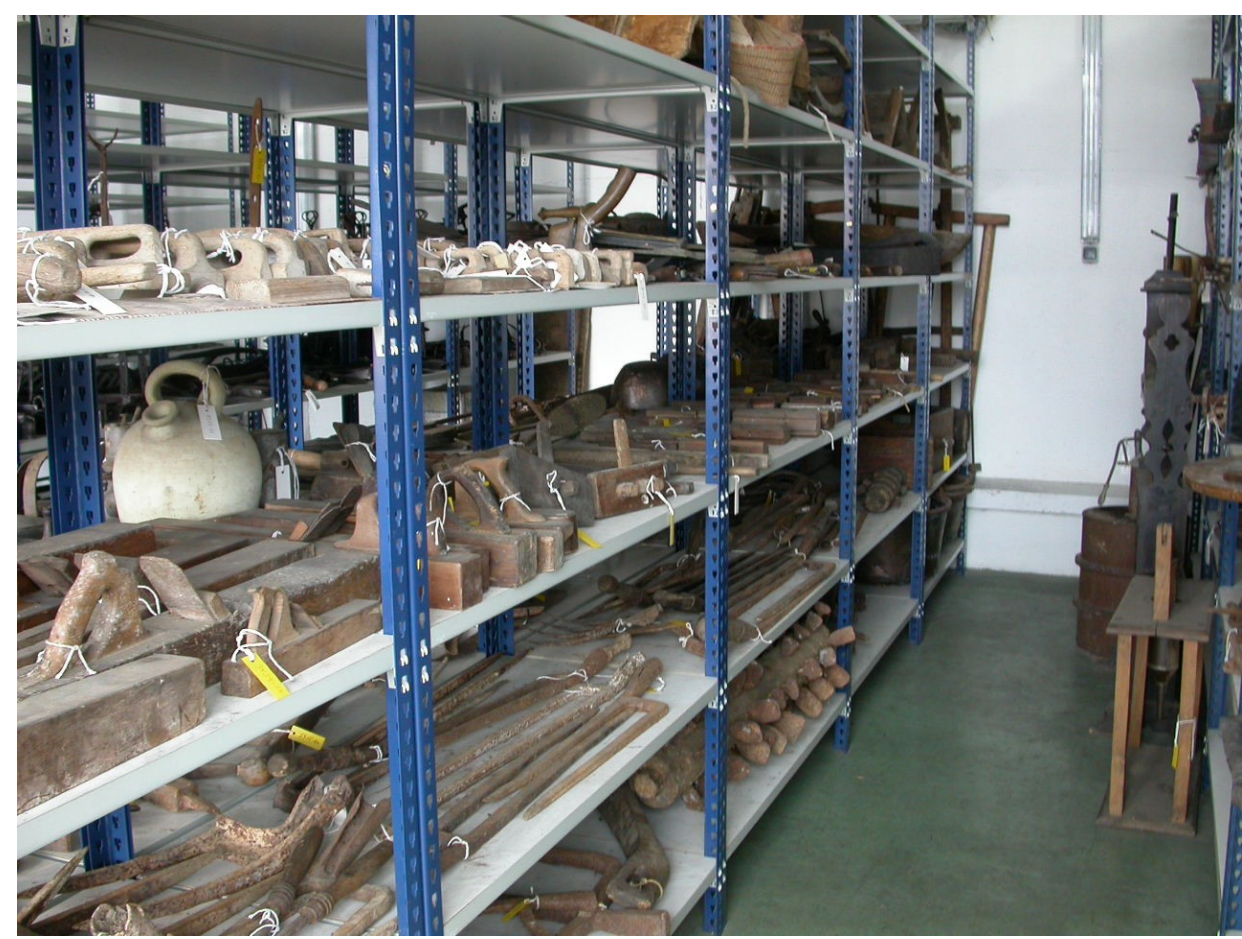

Figura 6: Imagen de los actuales depósitos de DFG.

A partir del registro de estos datos se procedió, en primer lugar, a ordenar los objetos por categorías climáticas ${ }^{6}$ :

I. Material etnográfico, lapídeo, madera, pintura/tela, pintura/tabla, escultura policromada, vidrio.

II. Papel, textil, cuero, piel y orgánico en general.

III. Objetos en metal, especialmente delicados.

En segundo lugar, se clasificaron por volúmenes y, seguidamente, por la superficie de ocupación, obteniendo el número de soportes necesarios para albergarlos. Es evidente que las medidas de los objetos y los formatos son variables, por lo que se han establecido unos estándares de mobiliario. $\mathrm{Al}$ resultado obtenido se le aplica un coeficiente corrector para poder sumar al área del objeto el espacio que necesita para su manipulación.

Así mismo, con los datos obtenidos, se han realizado propuestas de aprovechamiento de las estanterías en alzado, teniendo en cuenta que la altura libre en los depósitos va a ser de 3, $5 \mathrm{~m}$.

Esta primera aproximación ha permitido conocer el número de módulos de estantería y peines necesarios para albergar las actuales colecciones. A partir de esta extrapolación, una ingeniería especializada en logística de almacenes transformó los datos suministrados en estándares comerciales de mobiliario, distribuidos en el plano de planta, pretendiendo la optimización del espacio disponible. 


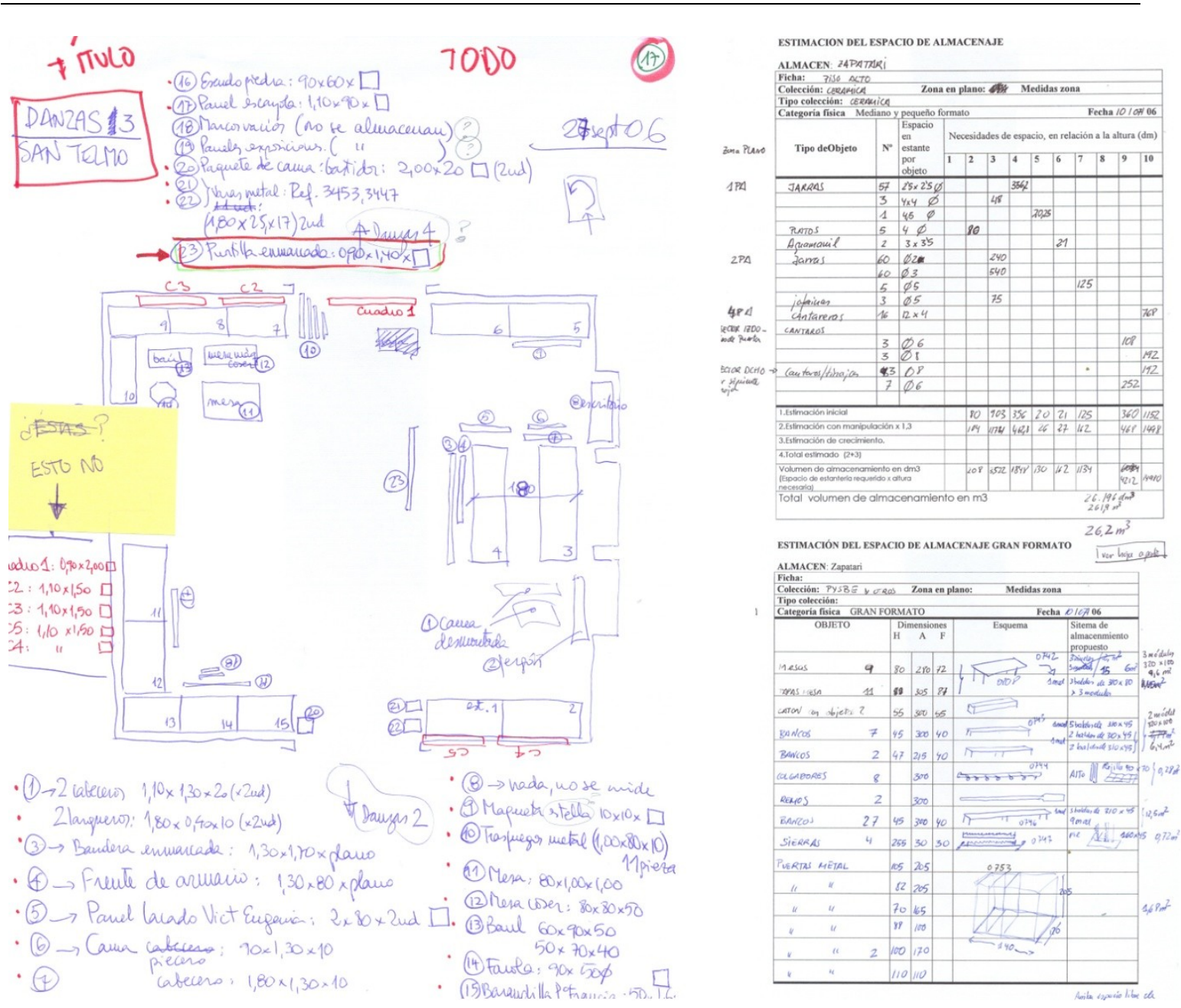

Figura 7: Análisis de colecciones, toma de datos.

La planificación de ciertas premisas de gestión de almacenaje, tales como la ubicación de los objetos de gran formato, o aquellos de más frecuente préstamo, en zonas de fácil acceso desde las vías de comunicación internas del edificio, también contribuirá a la hora de obtener este aprovechamiento máximo.

Así mismo, la distribución en planta del mobiliario permite al equipo redactor del proyecto arquitectónico realizar ciertas modificaciones en el diseño de instalaciones antes de la construcción. De esta manera, ciertos dispositivos de climatización, seguridad, accesos, etc. pueden ser ubicados con mayor facilidad, evitando interferencias no deseables con los elementos custodiados.

Por otra parte, la actualización y revisión de inventarios que están realizando tanto la Diputación Foral de Gipuzkoa como el Museo de San Telmo, dará lugar a un ajuste y precisión de los datos hasta ahora manejados. Gracias a ello, se conseguirá determinar con mayor exactitud las características del mobiliario, los sistemas de acondicionamiento necesarios y una distribución de los objetos en función de su naturaleza, volumen y masa, que permita una mejor gestión, control y conservación de los mismos.

Estos inventarios actualizados serán una herramienta básica a la hora de diseñar el traslado con garantías de los objetos desde su lugar de origen hasta el Centro, permitiendo una llegada ordenada y evitando colapsos en la recepción de los mismos. 


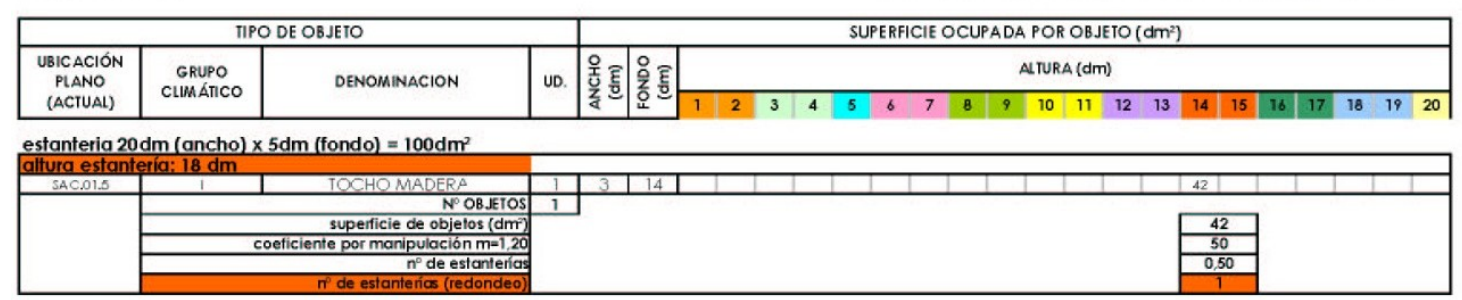
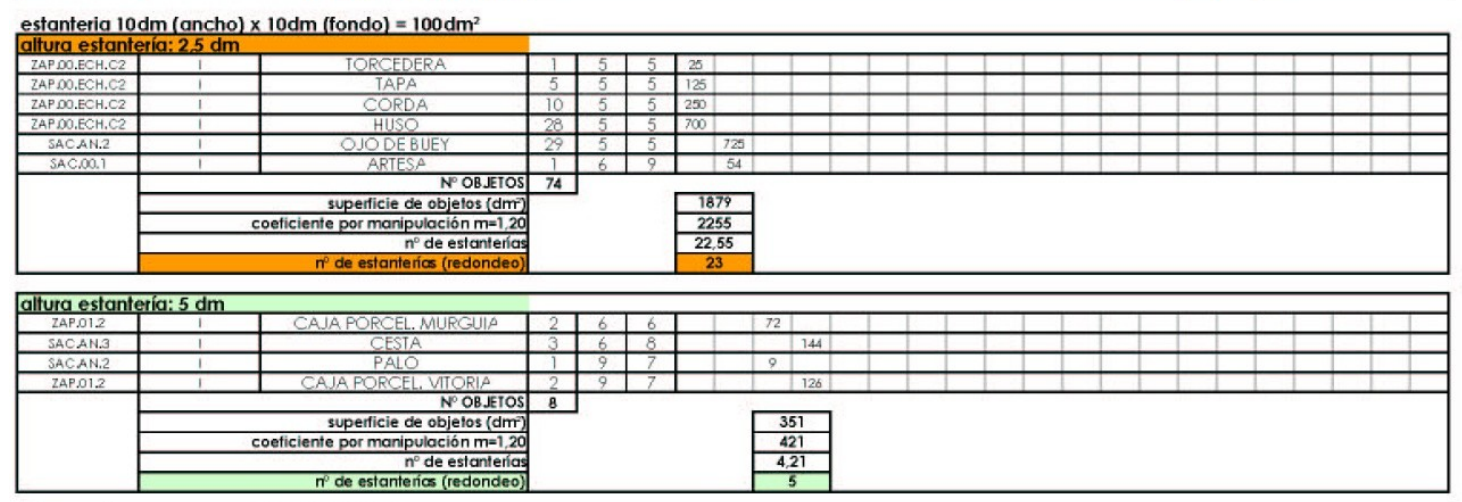

ALMACEN: DIPUTACION

Categoría física: PEQUEÑO Y MEDIANO FORMATO. OBJETOS SUELTOS

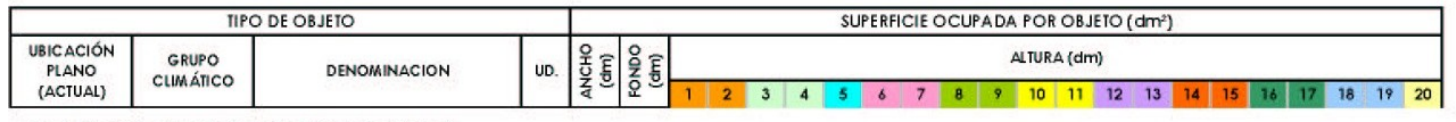

\begin{tabular}{|c|c|c|c|c|c|c|c|c|c|c|c|c|c|c|c|c|c|c|c|c|c|c|c|c|c|}
\hline Ifure & 50 & & & & & & & & & & & & & & & & & & & & & & & & \\
\hline 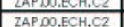 & $\frac{1}{1}$ & COLLAR & 50 & & & $\frac{1}{1}$ & $\frac{25}{100}$ & & & & & & & & & & & & & & & & & & \\
\hline \begin{tabular}{|l|}
$Z A P O O O, E C H, C 2$ \\
\end{tabular} & 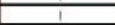 & LAMPARA ACEIIE & 81 & & & 1 & & 81 & & & & & & & & & & & & & & & & & \\
\hline \begin{tabular}{|l|} 
ZAPDOO.ECH.C2 \\
\end{tabular} & 1 & CANDELERO & 180 & & & 1 & & 180 & & & & 7 & 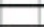 & 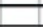 & - & 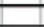 & & & & & 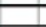 & +2 & - & 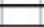 & \\
\hline \begin{tabular}{|l|} 
ZAP $00, E C H . C 2$ \\
\end{tabular} & 1 & BARBERIA & 15 & & & 2 & 30 & & & & & & & & & & & & & & & & & & \\
\hline \begin{tabular}{|l|} 
ZAPDOO.ECH.CZ \\
\end{tabular} & 1 & CATALEIC & 15 & & & 1 & 23 & & & & & & & & & & & & & & & & & & \\
\hline \begin{tabular}{|l|l|} 
ZAPDOO,ECH,C2 \\
\end{tabular} & $T$ & BOCALLAVE & 17 & & & 1 & 26 & & & & & & & & & & & & & & & & & & \\
\hline \begin{tabular}{|l|} 
ZAPDOO.ECH.C2 \\
\end{tabular} & $T$ & PASADOF & 46 & & & 1 & 46 & & & & & & & & & & & & & & & & & & \\
\hline \begin{tabular}{|l|} 
ZAPDOO.ECH.CZ \\
\end{tabular} & - & ISOPO ACEIRE & 13 & & & 1 & & 26 & & & & & & & & & & & & & & & & & \\
\hline \begin{tabular}{|l|l|}
$Z A P O O, E C H$ & \\
\end{tabular} & - & CANDIL & 60 & & & 1 & & $\frac{20}{120}$ & & & & & & & - & & & & & & & & & & \\
\hline \begin{tabular}{|l|} 
ZAPDOO.ECH.C2 \\
\end{tabular} & & ALMIREZ & 4 & & & 2 & & 16 & & & & & & & & & & & & & & & & & \\
\hline \begin{tabular}{|l|} 
ZAP $00 . E C H . C 2$ \\
\end{tabular} & 7 & RELOJ & 20 & & & 2 & & 80 & & & & & & & & & & & & & & & & & \\
\hline \begin{tabular}{|l|} 
ZAPDOO.ECH.C2 \\
\end{tabular} & 1 & REPOSTERIÁA & 40 & & & 2 & & 180 & & & & & & & & & & & & & & & & & \\
\hline \begin{tabular}{|l|} 
ZAP,OO.ECH,CZ2 \\
\end{tabular} & $T$ & FRONTIL & 6 & & & 4 & & 48 & & & & & & & & & & & & & & & & & \\
\hline \begin{tabular}{|l|} 
ZAPDOO.ECH.CZ \\
\end{tabular} & 1 & ARREO GANADERI/A & 13 & & & 4 & & 104 & & & & & & & & & & & & & & & & & \\
\hline SAC,AN,A & $I$ & VARIOS & 9 & & & 3 & 56 & & & & & & E & E & 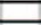 & E & E & - & & & & & & & \\
\hline SACAN.1 & & OBJETOS VARIOS & 73 & & & 3 & & 456 & & & & & & & & & & & & & & & & & \\
\hline \begin{tabular}{|l|} 
ZAP.0O.ECH.CZ \\
\end{tabular} & & PEROLO & 83 & & & 3 & & 519 & & & & & & & & & & & & & & & & & \\
\hline \begin{tabular}{|l|l|} 
ZAP OOO.ECH.C2 \\
\end{tabular} & & FAROL & 19 & & & 2 & & 86 & & & & & & & & & & & & & & & & & \\
\hline \begin{tabular}{|l|} 
ZAP OO. ECH.CZ \\
\end{tabular} & & HUSILLO & 4 & & & 2 & & 24 & & & & & & & & & & & & & & & & & \\
\hline \begin{tabular}{|l|}
$Z A P O O 0, E C H, C 2$ \\
\end{tabular} & & MORILLO & 22 & & & 2 & & 192 & & & & & & & & & & & & & & & & & \\
\hline 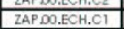 & 1 & TAPA & $\frac{22}{2}$ & & & $\frac{2}{3}$ & 18 & & & & & & & & & & & & & & & & & & \\
\hline \begin{tabular}{|l|}
$\angle A P O O, E C H, C 2$ \\
\end{tabular} & & TRANCA VENTANF & 2 & & & 3 & 18 & & & & & & & & & & & & & & & & & & \\
\hline \begin{tabular}{|l|} 
ZAPDOO.ECH.O1 \\
\end{tabular} & 1 & PASADOR DE COCINA & 6 & & & 3 & 54 & & & & & & & & & & & & & & & & & & \\
\hline \begin{tabular}{|l|} 
ZAP $00, E C H, C 2$ \\
\end{tabular} & 1 & PILAAGUABENDITA & 3 & & & 3 & & 27 & & & & & & & & & & & & & & & & & \\
\hline \begin{tabular}{|l|}
$Z A P Q 0, E C H, C 2$ \\
\end{tabular} & 1 & TREBEDE & 24 & & & 3 & & 216 & & & & & & & & & & & & & & & & & \\
\hline SAC.00.1 & I & & 1 & & & 2 & & 8 & & & & & & & & & & & & & - & 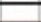 & 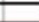 & 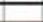 & \\
\hline \begin{tabular}{|l|} 
ZAP $D 0 ., E C H . H 13$ \\
\end{tabular} & I & CORTADORA & 8 & & & 3 & 90 & - & & & & & & & - & & & & & & & & & 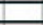 & \\
\hline \begin{tabular}{|l|} 
ZAPDOO.ECH.C2 \\
\end{tabular} & & MARMITA & 16 & & & 4 & 256 & & & & & & & & & & & & & & & & & & \\
\hline
\end{tabular}

Figura 8: Hojas de cálculo con los datos de los formularios de análisis de colecciones.

$3^{\circ}$ Condiciones Medioambientales

Dentro del análisis de riesgos previsibles para las colecciones depositadas en Gordailu, la implantación de las condiciones medioambientales adecuadas para la conservación de los objetos constituye uno de los elementos más problemáticos a la hora del diseño de la infraestructura. Al costo de inversión que implica una instalación de climatización, se añaden los gastos de explotación que pueden hipotecar el correcto funcionamiento del servicio que el Centro pretende dar. 
Por ello, a la hora del diseño del edifico, se ha hecho especial hincapié en la necesidad de conseguir la mayor inercia climática posible del mismo, con el fin de obtener un fácil mantenimiento de las consignas climáticas y lograr que las fluctuaciones que puedan producirse sean lentas y graduales.

De este modo, se pretende evitar tanto el sobredimensionamiento de los equipos de climatización como la continua dependencia de los mismos para conseguir el control climático.

El resultado de estas consideraciones previas ha sido una construcción de gran eficiencia energética, que busca, además, alternativas energéticas a través de una huerta solar fotovoltaica instalada en cubierta y un sistema de captación geotérmica. Gracias a esta iniciativa se va a conseguir de forma limpia el 80\% de la energía necesaria para la climatización7.

\section{Resultados}

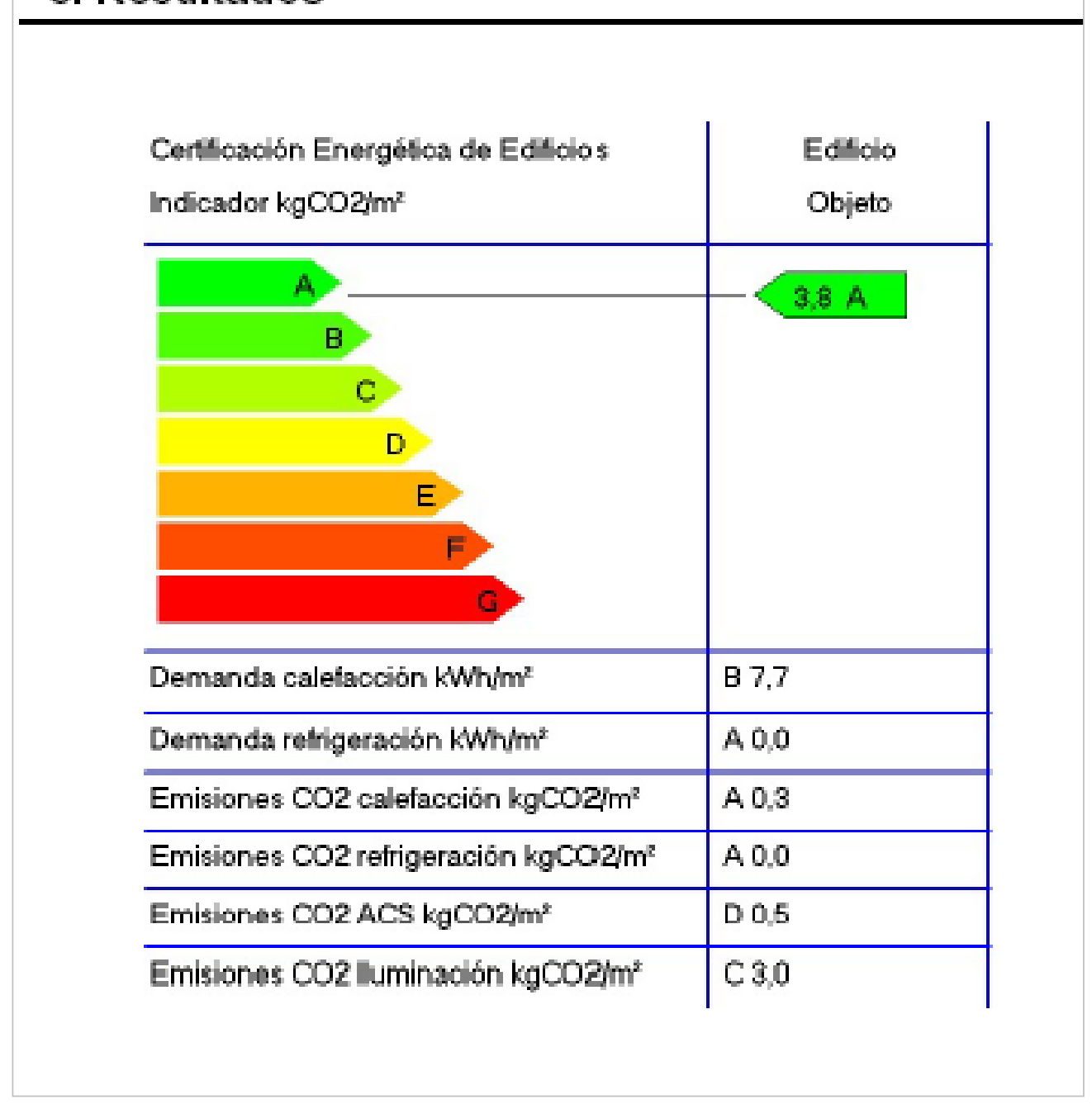

Figura 9: En el proyecto de arquitectura, se han definido las condiciones necesarias para obtener la calificación energética tipo A, que deberá ser objeto de una nueva certificación una vez finalizada la ejecución de la obra. Fuente: Astigarraga y Lasarte arquitectos. Proyecto de Ejecución.

En el mismo sentido, y con la finalidad de un uso racional de la climatización, se han establecido diferentes sectores climáticos, que permiten adecuar los diferentes espacios a sus necesidades reales. No debemos olvidar que más del 70\% del edificio (el dedicado a depósitos), no requiere estándares de confort humano, y que puede haber espacios con necesidades de flexibilidad climática que no deben afectar a otros. 
Para establecer estas consignas climáticas se analizaron, por una parte, la naturaleza de las colecciones a almacenar en Gordailu y su currículo climático. Por otra, las condiciones medioambientales del entorno del edificio. Todo ello con el fin de encontrar parámetros de compromiso que permitan una adecuada conservación de los objetos y un ahorro en los costos de climatización.

La mayoría de los objetos que van a depositarse en Gordailu se han conservado en almacenes donde las condiciones medioambientales no han podido ser controladas suficientemente. A pesar de que los niveles de humedad relativa no han sido en ocasiones los óptimos, en una primera evaluación, el porcentaje de objetos que presenta daños es reducido (datos de la Diputación Foral de Gipuzkoa y del Museo de San Telmo).

En cuanto al entorno del edificio, se han tomado como referencia básica los datos de la estación metereológica del aeropuerto de Hondarribia, situada a 2,8 km (Agencia Estatal de Meteorología, 2009; AAVV, 2005; Euskalmet, 2009).

Irún posee un clima templado húmedo. Las precipitaciones se distribuyen de forma homogénea a lo largo del ciclo anual, con ausencia de meses secos y máximo de lluvias en la época de otoñoinvierno. Las temperaturas son moderadas a lo largo de todo el año gracias a la cercanía del mar y al predominio de vientos sur-suroeste en invierno y norte-noroeste en verano. Las máximas se registran en julio-agosto y las mínimas en diciembre-enero. Las temperatura fluctúa desde $\operatorname{los} 9^{\circ} \mathrm{C}$ de media del mes de enero y los $21,5^{\circ} \mathrm{C}$ de agosto (medias mínimas $4,4^{\circ} \mathrm{C}$ y medias máximas $25,2^{\circ}$ C).

Diversos accidentes geográficos modulan el clima de la zona: la montaña de Jaizkibel actúa como protector ante los temporales, mientras que el macizo de Peña de Aia retiene la humedad y provoca una elevada pluviosidad. Las medias de humedad relativa son bastante elevadas y presentan una escasa variación a lo largo del año. Los valores medios mensuales registrados están comprendidos entre el 70,8\% del mes de marzo y el 75,6\% de los meses de agosto y noviembre. Los mínimos mensuales registrados corresponden a humedades relativas del 62\% (marzo de 1990), mientras los máximos alcanzan porcentajes del $90 \%$ (julio de 1983). A pesar de esta estabilidad, nos encontramos con un número importante de jornadas en las que la media supera el $95 \%$.

Considerando estos datos y la naturaleza de las colecciones, se han establecido unos parámetros medioambientales apropiados para la mayor parte de los objetos, teniendo en cuenta así mismo el concepto de fluctuación admisible a corto plazo y las variaciones estacionales.

Consigna general depósitos:

Humedad Relativa $=60 \%+/-5 \%$ (fluctuaciones de corta duración)

Temperatura $=20^{\circ} \mathrm{C}+/-2^{\circ} \mathrm{C}$

Fluctuación estacional $-5^{\circ} \mathrm{C}$

Esta consigna estará en vigor en la mayor parte de los espacios dedicados a almacenamiento. Para los objetos más vulnerables se establecen otros dos ámbitos diferenciados, además de utilizar contenedores herméticos especiales para aquellos objetos que lo requieran:

Espacio B:

Humedad Relativa $=50 \%+/-5 \%$

Temperatura $=20^{\circ} \mathrm{C}+/-2^{\circ} \mathrm{C}$

Fluctuación estacional $-5^{\circ} \mathrm{C}$

Espacio C:

Humedad Relativa $=40 \%+/-5 \%$

Temperatura $=20^{\circ} \mathrm{C}+/-2^{\circ} \mathrm{C}$ 
Fluctuación estacional - $5^{\circ} \mathrm{C}$

Para el control climático del edificio, además de las propias sondas de los equipos de climatización, se prevé instalar otro sistema en paralelo que permita una evaluación más exhaustiva de las condiciones climáticas.

El análisis del comportamiento del edificio durante el primer año permitirá realizar ajustes que conduzcan a una mayor eficacia en la consecución de los parámetros y una disminución del costo de explotación.

Otro factor importante a analizar en un espacio medioambientalmente controlado son los contaminantes del aire. Tras el análisis realizado a los datos recogidos en la estación de vigilancia y control de la calidad del aire de Irun ${ }^{9}$ Departamento de Medio Ambiente, Planificación Territorial, Agricultura y Pesca, 2009; Tetrault, 2003; Grzywacz, 2006), donde no se han detectado superaciones de los límites legales de los contaminantes medidos (CO, NO2, PM10, SO2 y O3), se ha constatado que la calidad del aire del entorno no es en principio problemática. No obstante, será necesario instalar los filtros pertinentes y establecer protocolos adecuados.

Por otra parte, los aerosoles marinos, fuente habitual de contaminación en instalaciones museísticas costeras de la provincia, no resultan tampoco preocupantes por la ubicación del edificio en una zona protegida de los vientos provenientes del mar gracias al monte Jaizkibel.

Una elección y gestión adecuada de los materiales que van a ser utilizados en la construcción y mobiliario de almacenamiento, así como una política de conservación preventiva en relación a la naturaleza de los objetos custodiados, contribuirá a rebajar la presencia de agentes contaminantes internos.

\section{Gordailu: aproximaciones a una gestión del nuevo equipamiento}

La puesta en marcha de un centro de estas características es un proceso complejo por si mismo, ya que la conservación y gestión de bienes culturales muebles implica muchos y distintos campos de actuación, tal y como hemos introducido en este artículo: desde el registro, inventariado, catalogación, documentación de diversa índole, hasta operaciones de conservación, manutención, almacenamiento, gestión de movimientos, préstamos, exposiciones, etc. A esta complejidad se añade la utilización coordinada de los depósitos por parte de instituciones diferentes y los proyectos de colaboración que entre ellas puedan surgir. Es por lo tanto imprescindible diseñar procedimientos y estándares que busquen la convergencia en un modelo y se consiga así un correcto funcionamiento de la infraestructura.

En este mismo sentido, y por centrarnos en las propias colecciones, antes de la apertura del Centro es necesario planificar la gestión de las mismas por parte de cada institución para su convergencia en Gordailu. En este apartado se agruparían todas aquellas operaciones necesarias para el correcto traslado de las colecciones y depósito de las mismas en el nuevo Centro, estableciendo criterios unificados y compartidos. En este campo se incluyen entre otros aspectos:

- Puesta a punto de las bases de datos de las diferentes colecciones y confluencia de las mismas para una evaluación exacta de sus características (agrupación de objetos por ámbitos climáticos, tipos de objetos, sistemas de almacenaje, volumen, fragilidad, acondicionamientos específicos, rotación, tratamientos requeridos, etc.).

- Definición y evaluación crítica de las colecciones con el fin de analizar su complementariedad, lagunas, patrones de crecimiento y posibles expurgos. 
- Implementación de un sistema definitivo de gestión informática integral.

- Preparación de las colecciones para el traslado desde sus actuales localizaciones, diseño y aplicación de estándares y procedimientos para su movimiento y depósito, acciones a realizar, diseño de cadenas de tratamiento, acondicionamiento. Circuitos y trazabilidad de los objetos. Cronogramas y definición de recursos materiales y humanos necesarios.

- Diseño del transporte y recepción de colecciones en Gordailu.

- Implementación de sistemas de señalización y siglado de objetos y localización en depósitos.

De la misma manera, se deberán implementar estándares de funcionamiento de los servicios del Centro. Incluyendo, entre otros, los relativos a la calidad y evaluación de los servicios, funcionamiento interinstitucional, seguridad, funcionamiento de depósitos (políticas de conservación preventiva, recepción y salida de objetos, embalaje, transporte, inventario y actualizaciones, préstamo, fotografía, conservación, revisión de fondos, manipulación, limpieza, etc), servicio de restauración, etc. También los referidos a la atención de público, visitas, centro de documentación, investigadores, exposiciones, actividades pedagógicas, información, difusión y comunicación, etc. Sin olvidar los parámetros generales de cualquier proyecto de creación de una organización de nuevo cuño y que no son el objeto de este artículo.

\section{Conclusión}

La creación de un Centro de estas características es un reto y un gran avance en materia de gestión de patrimonio. No sólo por el beneficio que supone para las propias colecciones su permanencia en una infraestructura adecuada para una correcta gestión de conservación, sino también por lo novedoso de la administración y usufructo de colecciones públicas (e incluso privadas) más allá de titularidades particulares, con el fin de conseguir la máxima accesibilidad a los fondos, su puesta en valor, así como el conocimiento y socialización de la importancia de la conservación y la implicación que debe tener la sociedad en este ámbito. Su próxima implantación podrá ser un ejemplo de actuación interinstitucional al servicio de todos los ciudadanos.

Albayalde SL. Empresa colaboradora en el proyecto.

Equipo:

Maite Barrio: Dirección

Ion Berasain: Coordinación

Benoit de Tapol: Consultor Conservación Preventiva

Amaia El Busto: Arquitecta

Imanol Mantiñan: Arquitecto

\section{Notas}

[1] El Plan de necesidades es un documento que define los aspectos generales (estructura, accesos, seguridad, comunicaciones, instalaciones generales, instalaciones), descripción de locales (por usos) necesarios para construir un edificio con un uso determinado, complementado por el estudio de la literatura técnica relacionada más reciente, así como una recopilación de la normativa específica de aplicación. 
[2] Para el estudio de espacios y su funcionamiento se han realizado visitas a diversos centros. Depósitos Museo del Traje, Museo del Prado, Museo Thyssen-Bornemisza, MNCARS, Casa de San Isidro, todos ellos en Madrid; MACBA, MNAC, Caixa Forum, Depósito arqueológico del Museo de la Ciudad. en Barcelona, Museo de Bellas Artes, Gugghenheim en Bilbao, Artium en Vitoria-Gasteiz. TEA, Valle de Guerra en Tenerife; Art et Metiers..en París; Réserves des Musées de Marseille, Marsella. Reservas externalizadas del Museo de Bellas artes y del Etnográfico de Perpiñan. Perpiñán, Victoria \& Albert, Science Museum y London Museum en Londres; National Conservation Centre en Liverpool, Glasgow Museum Resources Center. Glasgow.

[3] La configuración del espacio de los depósitos de pequeño y mediano permitirá albergar, en caso necesario, algunos objetos de gran volumen.

[4] Las características formales del solar y una comprensible contención presupuestaria hacen inevitable la revisión de las necesidades planteadas en el modelo ideal para poder materializarlo en el proyecto de ejecución. Siempre trabajando con la idea del buen funcionamiento del servicio y la optimización de espacio en vista a conseguir la mayor cantidad de superficie de almacenamiento posible.

[5] La metodología utilizada está basada en el estudio de Waltston y Bertram, 1992. 6, es decir, en función de los parámetros climáticos necesarios para una buena conservación de dichos objetos.

[7] La actuación prevista en el Centro Gordailu se incluye en el Programa para la sostenibilidad energética en Gipuzkoa, que contempla la mejora ambiental en el área del consumo energético, uno de cuyos objetivos es conseguir un suministro y consumo de energías más limpias aumentando el uso de energías renovables.

[9] Agradecemos a Unai Zeberio, técnico de control de aire del Departamento de Medio Ambiente, Planificación Territorial, Agricultura y Pesca, los datos suministrados. Estación de Irún, Avda. de la Aduana $\mathrm{n}^{\mathrm{o}} 28$.

\section{Bibliografía}

WALTSTON, W y BERTRAM, B. (1992). "Estimating space for the storage of ethnographic collections". En: Conservation-restauration des Biens Culturels Recherches et techniques Actuelles; La Conservation préventive. 3 Colloque de l'association des Restaurateurs d'Art et d'Archéologie de Formation Universitaire. Paris.

AGENCIA.ESTATAL.DE.METEOROLOGÍA:

http://www.aemet.es/es/elclima/datosclimatologicos/valoresclimatologicos?]=1014\&k=pva [consulta actualizada 5/6/2009]

AA.VV: (2005) Guía resumida del clima en España 1971-2004. Madrid: Ministerio de Medio Ambiente.

EUSKALMET (Euskal metereologia agentzia). Datos estaciones: http://www.euskalmet.euskadi.net/s07$5853 \mathrm{x} / \mathrm{es} / \mathrm{meteorologia} /$ lectur.apl?e=5\&amp;campo=C071-Jaizkibel [consulta actualizada 5/6/2009]

DEPARTAMENTO DE MEDIO AMBIENTE, PLANIFICACIÓN TERRITORIAL, AGRICULTURA Y PESCA, Red de vigilancia y control de calidad del aire: Datos históricos (últimos 10 años) http://www.ingurumena.ejgv.euskadi.net/r49-

3614/es/contenidos/informacion/historicos red aire/es red aire/indice.html [consulta actualizada 5/6/2009]. Red de vigilancia y control de calidad del aire: http://www.ingurumena.ejgv.euskadi.net/r493614/es/contenidos/informacion/red calida aire capv/es 975/indice calidad c.html [consulta actualizada $5 / 6 / 2009]$

TETRAulT, J. (2003). Airborne Pollutants in Museums, Galleries and Archives: Risk Assesment, Control Strategies and Preservation Managment. Ottawa: Canadian Conservation Institute.

GRZYWACZ, C.M. (2006) Monitoring for Gaseous Pollutants in Museums environments. .Los Ángeles: Getty Publications. 

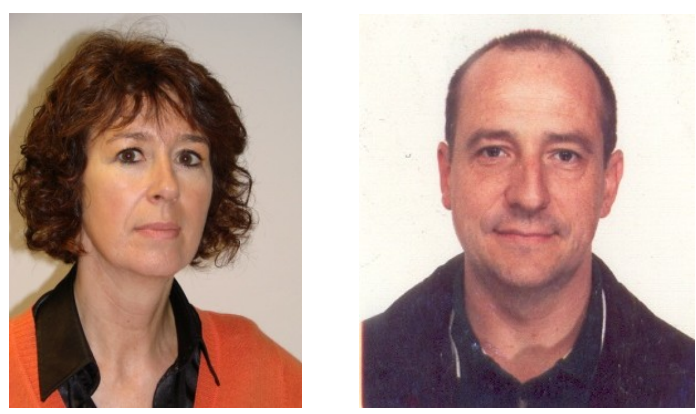

Maite Barrio \& Ion Berasain

Albayalde SL.

Río Deba 7, bajo 20012

San Sebastián.

albayalde@euskalnet.net

Maite Barrio y Ion Berasain son licenciados en $\mathrm{H}^{\mathrm{a}}$ del Arte, y Diplomados en Conservación y Restauración de obras de arte así como en Museología, siendo la primera, además, Licenciada en Ciencias Técnicas, especialidad conservación de bienes culturales. Su formación se ha realizado en diferentes universidades y centros españoles y extranjeros (Francia, Italia, Bélgica). Desde hace más de 20 años desarrollan su labor profesional en el campo del estudio, conservación, restauración y gestión de patrimonio. Han publicado numerosos artículos y monografías y han participado en congresos, jornadas y proyectos internacionales 